Internal labelling problem: an algorithmic procedure

This article has been downloaded from IOPscience. Please scroll down to see the full text article.

2011 J. Phys. A: Math. Theor. 44025204

(http://iopscience.iop.org/1751-8121/44/2/025204)

View the table of contents for this issue, or go to the journal homepage for more

Download details:

IP Address: 147.96.14.15

The article was downloaded on 15/04/2013 at 09:03

Please note that terms and conditions apply. 


\title{
Internal labelling problem: an algorithmic procedure
}

\author{
Rutwig Campoamor-Stursberg \\ Departamento Geometría y Topología, Fac. CC. Matemáticas, Universidad Complutense de \\ Madrid, Plaza de Ciencias 3, E-28040 Madrid, Spain \\ E-mail: rutwig@mat.ucm.es
}

Received 2 June 2010, in final form 22 October 2010

Published 9 December 2010

Online at stacks.iop.org/JPhysA/44/025204

\begin{abstract}
Combining the decomposition of Casimir operators induced by the embedding of a subalgebra into a semisimple Lie algebra with the properties of commutators of subgroup scalars, an analytical algorithm for the computation of missing label operators with the commutativity requirement is proposed. Two new criteria for subgroups scalars to commute are given. The algorithm is completed with a recursive method to construct orthonormal bases of states. As examples to illustrate the procedure, four labelling problems are explicitly studied.
\end{abstract}

PACS numbers: $02.20 \mathrm{~Sv}, 03.65 \mathrm{Fd}$

\section{Introduction}

One recurring problem often faced in physical applications of group theoretical methods is the appropriate choice of representation bases, whenever irreducible representations (IR) of a Lie group $G$ are decomposed with respect to some (physically relevant) subgroup $H$. It is not an uncommon phenomenon that the physically relevant basis is not sufficiently or adequately specified, resulting in some missing quantum numbers to specify the states without ambiguity [1]. One effective way, among the various different methods proposed in the literature to solve the so-called internal labelling or missing label problem (short MLP), consists of determining, within the enveloping algebra of the group $G$, a set of commuting subgroup scalars that are independent from the Casimir operators of $G$ and $H$. The common eigenstates of this complete set of Hermitian operators formed by the labelling operators, the Casimir operators of the groups and some appropriate internal subgroup operators can be chosen as the basis states for irreducible representations of $G$, the missing labels being specified by the eigenvalues of the labelling operators [2, 3]. However, explicit computations in the enveloping algebras of Lie algebras remain a difficult problem, and only a limited number of labelling problems have been completely solved using this direct technique. Although the general ansatz for internal labelling admits an analytical approach in terms of partial differential equations, the solutions of such systems are far from being trivially deduced, and the commutativity constraint of missing label operators is not entirely obvious. Recently an analytical criterion to check the 
commutativity of labelling operators of a certain type was developed [4], simplifying to some extent this additional requirement of the MLP.

The objective of this work is to enlarge some previous work on labelling operators (see e.g. $[4,5]$ and references therein) and to propose an algorithm to solve the labelling problem associated with semisimple Lie algebras ${ }^{1}$, based on the analytical approach. The algorithm consists of six main steps and is the result of combining and refining different approaches and extracting new criteria. Specifically, we take into account the decomposition of Casimir operators with respect to scale transformations induced by reduction chains (or embeddings of Lie algebras), which provide 'natural' candidates for the subgroup scalars, the criterion to check the commutativity of subgroup scalars in terms of Berezin brackets and various decomposition properties of commutators of labelling operators. Introducing a bi-degree for subgroup scalars, we obtain a procedure to construct commuting subgroup scalars, similar to that obtained in [6]. This step always simplifies the implementation of the algorithm. As a complement to the algorithm, we propose a direct generic procedure to construct an orthonormal basis of eigenstates for the (Hermitian) labelling operators, in the case of semisimple Lie algebras $\mathfrak{s}$ and $\mathfrak{s}^{\prime}$. The method is based on the successive diagonalization of the Casimir operators of the subalgebra $\mathfrak{s}^{\prime}$, the missing label operators and the internal subgroup operators, in that specific order. Orthonormalization follows by the usual techniques.

Four examples have been chosen to illustrate the algorithm and the basis construction: $G_{2} \supset \mathfrak{s u}(3)$ with one missing label, $\mathfrak{s o}(7) \supset[\mathfrak{s u}(2)]^{3}$ with $n=3$ missing labels, $\mathfrak{s u}(6) \supset \mathfrak{s o}(6)$ with $n=6$ labels and $\mathfrak{s o ( 7 )} \supset \mathfrak{s o ( 5 )}$ also with $n=3$ labels. For the first chain, we diagonalize the missing label operators and explicitly give a basis of eigenstates for the 64-dimensional IR of $G_{2}$. For the remaining three examples, we compute the missing label operators by means of the algorithm, and indicate how an orthonormal basis could be chosen. As far as the author is aware, it is the first time that the required missing label operators have been explicitly given for a generic IR in these cases. It should be remarked that the fourth labelling problem is structurally slightly different from the case $\mathfrak{s o}(7) \supset \mathfrak{s o}(5) \times \mathfrak{s o}(2)$, which is well known [7].

\section{Missing label operators}

It constitutes a classical result that any semisimple Lie algebra $\mathfrak{s}$ of rank $l$ possesses exactly $\mathcal{N}(\mathfrak{s})=l$ independent Casimir operators, i.e. polynomials in the generators that commute with all elements of the algebra. The eigenvalues of Casimir operators can be used to label irreducible representations (IRs) of $\mathfrak{s}$, while the Cartan subalgebra identifies states within a multiplet. However, usually these operators are not enough to completely separate multiplicities, and additional operators must be determined. It can be shown that the total number of internal labels required is

$$
\mathrm{i}=\frac{1}{2}(\operatorname{dim} \mathfrak{s}-\mathcal{N}(\mathfrak{s})) \text {. }
$$

The same pattern is observed when a subalgebra $\mathfrak{s}^{\prime}$ is used to label the basis states of irreducible representations of $\mathfrak{s}$. The subgroup provides $\frac{1}{2}\left(\operatorname{dim} \mathfrak{s}^{\prime}+\mathcal{N}\left(\mathfrak{s}^{\prime}\right)\right)-l_{0}$ labels, where $l_{0}$ is the number of invariants of $\mathfrak{s}$ that depend only on generators of the subalgebra $\mathfrak{s}^{\prime}$ [8]. To distinguish elements within an IR of $\mathfrak{s}$, we need to find

$$
n=\frac{1}{2}\left(\operatorname{dim} \mathfrak{s}-\mathcal{N}(\mathfrak{s})-\operatorname{dim} \mathfrak{s}^{\prime}-\mathcal{N}\left(\mathfrak{s}^{\prime}\right)\right)+l_{0}
$$

additional operators, called missing label operators or subgroup scalars. The total number of available operators of this kind is easily shown to be twice the number of needed labels, i.e. $m=2 n$. For $n>1$, the labelling operators must moreover commute with each other.

1 It can be generalized to direct sums of semisimple and Abelian, i.e. reductive algebras, in a straightforward manner. 
For this type of state labelling, the Casimir operators of $\mathfrak{s}$ are used to characterize the irreducible representations $R$, while the (missing) labelling operators and the internal subgroup operators will separate degeneracies and distinguish states within the IRs of $\mathfrak{s}^{\prime}$ appearing in $R$. Typically, these internal subgroup operators consist of the Cartan generators and Casimir operators of $\mathfrak{s}^{\prime}$, as well as some additional Casimir operators of other subalgebras canonically embedded into $\mathfrak{s}^{\prime}[1,2]$.

The similarities between the internal labelling problem and the determination of invariants of Lie algebras allow one to adapt the analytical approach [8]. If $\mathfrak{s}$ is a Lie algebra with generators $\mathbb{R}\left\{X_{1}, \ldots, X_{n}\right\}$ and commutators $\left[X_{i}, X_{j}\right]=C_{i j}^{k} X_{k}$, the $X_{i}$ 's are realized in the space $C^{\infty}\left(\mathfrak{s}^{*}\right)$ by means of the differential operators:

$$
\widehat{X}_{i}=C_{i j}^{k} x_{k} \frac{\partial}{\partial x_{j}},
$$

where $\left\{x_{1}, \ldots, x_{n}\right\}$ are the coordinates of a covector in a dual basis of $\mathbb{R}\left\{X_{1}, \ldots, X_{n}\right\}$. The invariants of $\mathfrak{s}$ (in particular, the Casimir operators) are then solutions of the following system of partial differential equations:

$$
\widehat{X}_{i} F=0, \quad 1 \leqslant i \leqslant n .
$$

The number $\mathcal{N}(\mathfrak{s})$ of functionally independent solutions of (4) is obtained from the classical criteria:

$$
\mathcal{N}(\mathfrak{s}):=\operatorname{dim} \mathfrak{s}-\sup _{x_{1}, \ldots, x_{n}} \operatorname{rank}\left(C_{i j}^{k} x_{k}\right),
$$

where $\left(C_{i j}^{k} x_{k}\right)$ is the matrix associated with the commutator table of $\mathfrak{s}$ over the given basis. For a polynomial solution of (4), the standard symmetrization map defined by

$$
\Lambda\left(x_{i_{1}} \ldots x_{i_{p}}\right)=\frac{1}{p !} \sum_{\sigma \in S_{p}} X_{\sigma\left(i_{1}\right)} \ldots X_{\sigma\left(i_{p}\right)}
$$

allows us to recover the Casimir operators in their usual form, i.e. as elements in the centre of the enveloping algebra $\mathcal{U}(\mathfrak{s})$. In particular, for any homogeneous polynomial $f=c^{k_{1} \ldots k_{p}} x_{k_{1}} \ldots x_{k_{p}}$ we can define uniquely its symmetric representative as

$$
\Lambda(f)=c^{k_{1} \ldots k_{p}} \Lambda\left(x_{k_{1}} \ldots x_{k_{p}}\right)
$$

Equation (7) provides an extension of the symmetrization map $\Lambda: S(\mathfrak{s}) \rightarrow \mathcal{U}(\mathfrak{s})$, where $S(\mathfrak{s})$ denotes the space of polynomials defined on the dual space $\mathfrak{s}^{*}$. Conversely, given a polynomial $P=c^{k_{1} \ldots k_{p}} X_{k_{1}} \ldots X_{k_{p}} \in \mathcal{U}(\mathfrak{s})$, we find its analytical counterpart $\pi(P)=c^{k_{1} \ldots k_{p}} x_{k_{1}} \ldots x_{k_{p}}$ by simply replacing the generator $X_{i}$ by the corresponding coordinates $x_{i}$ of $\mathfrak{s}^{*}$. In this context, two monomials $P=X_{i_{1}} \ldots X_{i_{p}} \in \mathcal{U}(\mathfrak{s}), Q=X_{j_{1}} \ldots X_{j_{q}} \in \mathcal{U}(\mathfrak{s})$ such that $\pi(P) \neq \pi(Q)$ are called factorizable if they can be written in the form

$$
P=X_{1}^{a_{1}} \ldots X_{l}^{a_{l}} P_{1} \in U(\mathfrak{s}), \quad Q=X_{l}^{a_{l}} \ldots X_{1}^{a_{1}} Q_{1} \in U(\mathfrak{s}),
$$

where $\left[P_{1}, Q_{1}\right]=0$ and $\left[X_{i}, P_{1}\right]=\left[X_{i}, Q_{1}\right]=0, i=1, \ldots, l$. The pair $P, Q$ is nonfactorizable if no decomposition of the preceding type exists (see [4]). In a straightforward generalization, two polynomials $F=c^{k_{1} \ldots k_{p}} X_{k_{1}} \ldots X_{k_{p}}$ and $G=c^{j_{1} \ldots j_{q}} X_{j_{1}} \ldots X_{j_{q}}$ are a nonfactorizable pair if for any pair $\left\{c^{k_{1} \ldots k_{p}}, c^{j_{1} \ldots j_{q}}\right\}$ the monomials $\left\{X_{k_{1}} \ldots X_{k_{p}}, X_{j_{1}} \ldots X_{j_{q}}\right\}$ do not admit a decomposition of type (8). Observe that taking the projection, this means that the terms share a common factor. In the following, we will use the term of non-factorizable pairs for both the symmetric and analytical representatives, whenever there is no ambiguity.

If we now consider the missing label problem associated with the embedding of Lie algebras $f: \mathfrak{s}^{\prime} \rightarrow \mathfrak{s}$, the missing label operators can be computed considering the equations of (4) corresponding to the generators of the subalgebra $\mathfrak{s}^{\prime}$. This system, as proven in [8], has 
exactly $\mathcal{N}\left(f\left(\mathfrak{s}^{\prime}\right)\right)=2 n+\mathcal{N}(\mathfrak{s})+\mathcal{N}\left(\mathfrak{s}^{\prime}\right)-l_{0}$ independent solutions, showing that the equations corresponding to subalgebra generators have $n$ more solutions as needed to solve the labelling problem.

\subsection{Decomposing Casimir operators of semisimple Lie algebras}

Any reduction chain $\mathfrak{s}^{\prime} \subset \mathfrak{s}$ induces branching rules of representations. In particular, the adjoint representation of $\mathfrak{s}$ decomposes as

$$
\operatorname{ad}(\mathfrak{s})=\operatorname{ad}\left(\mathfrak{s}^{\prime}\right) \oplus R,
$$

where $R$ is a (completely reducible) representation of $\mathfrak{s}^{\prime} .^{2}$ The latter equation reflects that we can always construct a basis of $\mathfrak{s}$ starting from an arbitrary basis of $\mathfrak{s}^{\prime}$ and taking into account how the remaining generators are coupled with those of the subalgebra (determined by the decomposition of $R$ into IRs of $\left.\mathfrak{s}^{\prime}\right)$.

The branching rule (9) can also be used to construct subgroup scalars for the corresponding MLP, as first developed in another context in [5]. For a non-zero constant $\varepsilon$ consider the linear isomorphism $\Phi: \mathfrak{s} \rightarrow \mathfrak{s}$ defined by

$$
\Phi\left(X_{i}\right)=\left\{\begin{array}{ll}
X_{i}, & 1 \leqslant i \leqslant n_{0} \\
\varepsilon X_{i}, & n_{0}+1 \leqslant i \leqslant n
\end{array},\right.
$$

where $\mathbb{R}\left\{X_{1}, \ldots, X_{n_{0}}, \ldots, X_{n}\right\}$ is a basis of $\mathfrak{s}$ such that $\mathbb{R}\left\{X_{1}, \ldots, X_{n_{0}}\right\}$ generates the subalgebra $\mathfrak{s}^{\prime}$ and $\mathbb{R}\left\{X_{n_{0}+1}, \ldots, X_{n}\right\}$ is a basis of $R .{ }^{3}$ If now $C_{p}=\kappa^{i_{1} \ldots i_{p}} X_{i_{1}} \ldots X_{i_{p}}$ is a Casimir operator of degree $p$ of $\mathfrak{s}$ (taken in its symmetric form), the expression of the transformed operator is given by

$$
C_{p}\left(\Phi^{-1}(\varepsilon)\left(X_{i_{1}}\right), \ldots, \Phi^{-1}(\varepsilon)\left(X_{i_{p}}\right)\right)=\varepsilon^{-\left(n_{i_{1}}+\cdots+n_{i_{p}}\right)} \kappa^{i_{1} \ldots i_{p}} X_{i_{1}} \ldots X_{i_{p}} .
$$

Now, defining $M_{p}=\max \left\{n_{i_{1}}+\cdots+n_{i_{p}} \mid \kappa^{i_{1} \cdots i_{p}} \neq 0\right\}$, the Casimir operator can be formally rewritten as a polynomial in $\varepsilon$ :

$$
C_{p}=\sum_{\alpha=0}^{M_{p}} \varepsilon^{\alpha} \Theta^{[p-\alpha, \alpha]},
$$

where $M_{p} \leqslant p$ and $\Theta^{[p-\alpha, \alpha]}$ is a homogeneous polynomial of degree $p-\alpha$ in the generators of the subalgebra $\mathfrak{s}^{\prime}$ and degree $\alpha$ in those associated with the tensor components of $R$. We shall say that $\Theta^{[p-\alpha, \alpha]}$ is an operator of bi-degree $(p-\alpha, \alpha)$. Since we are only interested in the resulting operators of (12) and not on the special value of $\varepsilon$, we take $\varepsilon=1$ and simply write $C_{p}=\sum_{\alpha=0}^{M_{p}} \Theta^{[p-\alpha, \alpha]}$. In the following we will use (12) with this abuse of notation. The interesting fact is that for any generator $X_{i} \in \mathfrak{s}^{\prime}$ the decomposition implies that $\left[X_{i}, \Theta^{[p-\alpha, \alpha]}\right]=0$. This is best seen taking the analytical approach. The analytical counterpart of (12) is easily found: $\pi\left(C_{p}\right)=\sum_{\alpha=0}^{M_{p}} \pi\left(\Theta^{[p-\alpha, \alpha]}\right)$. The differential operator associated with the generator $X_{i}$, applied to $\pi\left(\Theta^{[p-\alpha, \alpha]}\right)$, is

$$
\widehat{X}_{i} \pi\left(\Theta^{[p-\alpha, \alpha]}\right)=C_{i j}^{k} x_{k} \frac{\partial \pi\left(\Theta^{[p-\alpha, \alpha]}\right)}{\partial x_{j}} .
$$

Since $\left[X_{i}, \mathfrak{s}^{\prime}\right] \in \mathfrak{s}^{\prime}$ and $\left[X_{i}, R\right] \in R$, the result of (13) is again a homogeneous polynomial of the same bi-degree as $\pi\left(\Theta^{[p-\alpha, \alpha]}\right)$. This means that evaluating $C_{p}$ gives rise to a sum of

2 Complete reducibility is actually ensured only if the subalgebra $\mathfrak{s}^{\prime}$ is semisimple.

3 These transformations and the subsequent decomposition of Casimir operators follow naturally from the approach to the MLP by means of contractions of Lie algebras [5]. 
polynomials of different bi-degree, and since $C_{p}$ is a Casimir operator, the only possibility is that each term is a solution of the system. This proves that the $\Theta^{[p-\alpha, \alpha]}$ are subgroup scalars.

The precise number of independent terms obtained from (12) depends on the representation $R$ induced by the reduction, although for any Casimir operator of degree $d \geqslant 3$ at least one independent operator is obtained. In many cases, however, more than one term of the same degree can be chosen. This is known to occur in various relevant labelling problems [9].

It is clear from the homogeneity property that linear combinations of the $\Theta^{[p-\alpha, \alpha]}$ and their commutators are themselves labelling operators [10]. In particular they can be expressible in terms of the elementary subgroup scalars. However, the decomposition (12) does not either guarantee that the relevant labelling operators must be functions of the $\Theta^{[p-\alpha, \alpha]}$ or that the labelling operators obtained by this procedure are mutually commutative. In some special circumstances, however, the decomposition (12) can provide commutativity directly. If for a specific MLP it is known that no solutions of bi-degree $(r, s)$ exist for some fixed $r+s=p+q$, and if we have two labelling operators such that $\left[\Theta^{\left[p-\alpha_{j}, \alpha_{j}\right]}, \Theta^{\left[q-\alpha_{k}, \alpha_{k}\right]}\right]$ is a sum of polynomials of bi-degree $(s, r)$, then the commutation follows at once. This idea was explored systematically in [6]. We remark that in the commutative frame, it would suffice to show that no polynomial function of bi-degree $(r, s)$ is a solution to the equations corresponding to subalgebra generators.

\section{Berezin brackets of labelling operators}

In this section we recall the notion of Berezin brackets applied to labelling operators, as recently developed in [4], and use these results to propose some new criteria to analyse the decomposition of commutators of labelling operators into sums of operators of certain bi-degrees.

The problem of finding a polynomial $f$ in $S(\mathfrak{s})$, the symmetrization $\Lambda(f)$ of which coincides with the commutator in $\mathcal{U}(\mathfrak{s})$ of two previously given symmetrized polynomials $\Lambda(g)$ and $\Lambda(h)$, was satisfactorily solved by Berezin in [11]. Given two (homogeneous) polynomials $g, h \in S(\mathfrak{s})$, the commutator $[\Lambda(g), \Lambda(h)]=\Lambda(g) \Lambda(h)-\Lambda(h) \Lambda(g)$ was shown to coincide with the symmetrization $\Lambda(f)$ of the polynomial

$$
f=-C_{i j}^{k} x_{k} \frac{\partial g}{\partial x_{i}} \frac{\partial h}{\partial x_{j}}+F\left(x_{k}, \cdots, \frac{\partial^{d} g}{\partial x_{j_{1}} \ldots \partial x_{j_{d}}}, \frac{\partial^{d} h}{\partial x_{j_{1}} \ldots \partial x_{j_{d}}}, \cdots\right),
$$

where $F$ is a polynomial, the terms of which involve derivatives of order $d \geqslant 2$ of $g$ and $h$ [see [11], equation (31)]. If $g, h$ are homogeneous of degrees $p$ and $q$, respectively, then $F$ decomposes as a sum of homogeneous polynomials of degrees $\leqslant p+q-2$ [11]. This enables us to rewrite the commutator as

$$
[\Lambda(g), \Lambda(h)]=\Lambda(\{g, h\})+\text { L.O.T. }
$$

where

$$
\{g, h\}=-C_{i j}^{k} x_{k} \frac{\partial g}{\partial x_{i}} \frac{\partial h}{\partial x_{j}}
$$

is the Berezin bracket of $g$ and $h$. The lower order terms correspond to the symmetric representative of $F[4,11]$. With the help of this bracket, an analytical criterion for two polynomials in the enveloping algebra $\mathcal{U}(\mathfrak{s})$ of a semisimple Lie algebra to commute was given in [4]: 
Theorem 1. Let $F, G$ be a non-factorizable pair of polynomials in the enveloping algebra $\mathcal{U}(\mathfrak{s})$ of $\mathfrak{s}$ such that $F=\Lambda(f), G=\Lambda(g)$ for some homogeneous polynomials $f, g \in S(\mathfrak{s})$. Then $[F, G]=0$ if and only if $\{f, g\}=0$, i.e. if the functions $f, g$ are in involution with respect to the Berezin bracket.

For the case of labelling operators, the preceding result provides a practical procedure to determine whether their symmetrizations commute or not. As a further consequence of equation (15) and the uniqueness of the symmetric representative of $\Lambda(f)$ for homogeneous polynomials $f \in S(\mathfrak{s})$, we can determine how a commutator $[\Lambda(g), \Lambda(h)]$ decomposes into a sum of labelling operators, as well as compute the bi-degree of the components.

In the following, we will always suppose that $\mathfrak{s} \supset \mathfrak{s}^{\prime}$ is a chain of semisimple Lie algebras ${ }^{4}$.

Lemma 1. Let $\Theta^{[p, q]}$ be an operator of bi-degree $(p, q)$. Then

$$
\left[\Theta^{[p, q]}, \Theta^{[r, s]}\right]=\Theta^{[p+r-1, q+s]}+\Theta^{[p+r, q+s-1]}+\Theta^{[p+r+1, q+s-2]} .
$$

The proof follows using the analytical counterpart of the operators. Let $\mathbb{R}\left\{X_{i}, T_{j}\right\}$ be a basis of $\mathfrak{s}$ such that the $X_{i}$ 's generate the subalgebra $\mathfrak{s}^{\prime}$ and the generators $T_{j}$ transform under $\mathfrak{s}^{\prime}$ like the representation $R$ of (9). Over such a basis the commutators have the form

$$
\left[X_{i}, X_{j}\right]=C_{i j}^{k} X_{k},\left[X_{i}, T_{j}\right]=\widehat{C}_{i j}^{k} T_{k},\left[T_{i}, T_{j}\right]=D_{i j}^{k} X_{k}+\widehat{D}_{i j}^{k} T_{k}
$$

Let $\left\{x_{i}, t_{j}\right\}$ be the corresponding coordinates in $\mathfrak{s}^{*}$. If $\Theta^{[p, q]}$ is an operator of bi-degree $(p, q)$, its projection by $\pi$ has the following form:

$$
\pi\left(\Theta^{[p, q]}\right)=O^{[p, q]}=\lambda^{i_{1} \ldots i_{p} j_{1} \ldots j_{q}} x_{i_{1}} \ldots x_{i_{p}} t_{j_{1}} \ldots t_{j_{q}} .
$$

The Berezin bracket of $O^{[p, q]}$ and $O^{[r, s]}$ is given by

$$
\begin{aligned}
\left\{O^{[p, q]}, O^{[r, s]}\right\} & =-\sum_{i<j} C_{i j}^{k} x_{k}\left(\frac{\partial O^{[p, q]}}{\partial x_{i}} \frac{\partial O^{[r, s]}}{\partial x_{j}}-\frac{\partial O^{[p, q]}}{\partial x_{j}} \frac{\partial O^{[r, s]}}{\partial x_{i}}\right) \\
& -\sum_{i<j} \widehat{C}_{i j}^{k} t_{k}\left(\frac{\partial O^{[p, q]}}{\partial x_{i}} \frac{\partial O^{[r, s]}}{\partial t_{j}}-\frac{\partial O^{[p, q]}}{\partial t_{j}} \frac{\partial O^{[r, s]}}{\partial x_{i}}\right) \\
& -\sum_{i<j}\left(D_{i j}^{k} x_{k}+\widehat{D}_{i j}^{k} t_{k}\right)\left(\frac{\partial O^{[p, q]}}{\partial t_{i}} \frac{\partial O^{[r, s]}}{\partial t_{j}}-\frac{\partial O^{[p, q]}}{\partial t_{j}} \frac{\partial O^{[r, s]}}{\partial t_{i}}\right) .
\end{aligned}
$$

It follows at once from this expression that the two first sums give rise to a homogeneous polynomial of bi-degree $(p+r-1, q+s)$, while the last sum splits into two homogeneous polynomials, the first having bi-degree $(p+r+1, q+s-2)$ and the second $(p+r, q+s-1)$. Taking the symmetric representatives of these polynomials by $\Lambda$, the assertion is proven. We remark that (17) does not exclude the possibility that some of the operators is zero.

The previous formula can be generalized in a straightforward manner to subalgebras of the type $\mathfrak{s}^{\prime}=\mathfrak{s}_{1} \oplus \ldots \oplus \mathfrak{s}_{n}$, in the case we want to distinguish the degree of the operators in each of the component subalgebras of $\mathfrak{s}^{\prime}$. If $\Theta^{\left[p_{1}, \ldots, p_{n} ; q\right]}$ denotes a subgroup scalar having degree $p_{i}$ in the generators of $\mathfrak{s}_{i}$ and degree $q$ in the components of $R$, then

$$
\begin{aligned}
{\left[\Theta^{\left[p_{1}, \ldots, p_{n} ; q\right]}, \Theta^{\left[r_{1}, \ldots, r_{n} ; s\right]}\right]=\sum_{\alpha=1}^{n} \Theta^{\left[p_{1}+r_{1}, \ldots, p_{\alpha}+r_{\alpha}-1, \ldots, p_{n}+r_{n} ; q+s\right]} } \\
\\
+\sum_{\alpha=1}^{n} \Theta^{\left[p_{1}+r_{1}, \ldots, p_{\alpha}+r_{\alpha}+1, \ldots, p_{n}+r_{n} ; q+s-2\right]}+\Theta^{\left[p_{1}+r_{1}, \ldots, p_{n}+r_{n} ; q+s-1\right]} .
\end{aligned}
$$

\footnotetext{
4 This requirement of semisimplicity is imposed to guarantee the existence of a complete basis of labelling operators
} formed by polynomials. It can however be generalized to reductive algebras. 
We observe that if the representation $R$ of (9) is such that $[R, R] \subset \mathfrak{s}^{\prime}$, then the last term on the right-hand side of (18) vanishes. Moreover, for operators with $p_{i}=r_{i}=0$, the first sum in (18) is also identically zero.

\subsection{Properties of commutators of subgroup scalars}

The preceding decomposition formula (17) can be further used to analyse the precise structure of the resulting scalars, as well as to establish some criteria for the commutativity of labelling operators. These will be useful to reduce the number of Berezin brackets that must be computed in labelling problems.

Suppose that $C_{p}$ is a Casimir operator that decomposes, with respect to the transformations (10), like

$$
C_{p}=\Theta^{[p-\alpha, \alpha]}+\Theta^{[p-\beta, \beta]},
$$

where $\alpha \neq \beta \neq 0$. Since $C_{p}$ commutes with any generator $X$ of $\mathfrak{s}$, it follows at once that $\left[\Theta^{[p-\alpha, \alpha]}, \Theta^{[p-\beta, \beta]}\right]=0$. One may ask whether this decomposition implies some relation between the indices $\alpha$ and $\beta$. To this extent, let $X$ be a generator of $\mathfrak{s}$ not belonging to $\mathfrak{s}^{\prime}$. Since $C_{p}$ is a Casimir operator of $\mathfrak{s}$, we have

$$
\left[X, C_{p}\right]=\left[X, \Theta^{[p-\alpha, \alpha]}\right]+\left[X, \Theta^{[p-\beta, \beta]}\right]=0 .
$$

Assuming that $X$ does not commute with the components of $C_{p}$, and according to (17), we obtain the commutator decomposition

$$
\begin{aligned}
& {\left[X, \Theta^{[p-\alpha, \alpha]}\right]=\Theta_{\alpha}^{[p-\alpha-1, \alpha+1]}+\Theta_{\alpha}^{[p-\alpha, \alpha]}+\Theta_{\alpha}^{[p-\alpha+1, \alpha-1]},} \\
& {\left[X, \Theta^{[p-\beta, \beta]}\right]=\Theta_{\beta}^{[p-\beta-1, \beta+1]}+\Theta_{\beta}^{[p-\beta, \beta]}+\Theta_{\beta}^{[p-\beta+1, \beta-1]},}
\end{aligned}
$$

where $X$ is considered as an operator of bi-degree $(0,1)$. Because of the homogeneity property, those operators, the bi-degree of which appears only once in the sum of (22a) and (22b), must vanish, while those having the same bi-degree must sum up to zero without being themselves zero. Essentially, the analysis of the bi-degree of the scalars leads to three possibilities for the value of $|\beta-\alpha|$.

(1) $\beta=\alpha+1$. From (22b) we obtain

$$
\left[X, \Theta^{[p-\beta, \beta]}\right]=\Theta_{\alpha+1}^{[p-\alpha-2, \alpha+2]}+\Theta_{\alpha+1}^{[p-\alpha-1, \alpha+1]}+\Theta_{\alpha+1}^{[p-\alpha, \alpha]} .
$$

Using the homogeneity property implies in particular that $\Theta_{\alpha+1}^{[p-\alpha-2, \alpha+2]}=0$, $\Theta_{\alpha}^{[p-\alpha-1, \alpha+1]}=0, \Theta_{\alpha}^{[p-\alpha, \alpha]}+\Theta_{\alpha+1}^{[p-\alpha, \alpha]}=0$ and $\Theta_{\alpha}^{[p-\alpha-1, \alpha+1]}+\Theta_{\alpha+1}^{[p-\alpha-1, \alpha+1]}=0$ at least one of the two last sums being satisfied non-trivially.

(2) $\beta=\alpha+2$. In this case, the right-hand side of $(22 b)$ reads

$$
\left[X, \Theta^{[p-\beta, \beta]}\right]=\Theta_{\alpha+2}^{[p-\alpha-2, \alpha+3]}+\Theta_{\alpha+1}^{[p-\alpha-2, \alpha+2]}+\Theta_{\alpha+1}^{[p-\alpha-1, \alpha+1]} .
$$

By the assumption, we must have $\Theta_{\alpha}^{[p-\alpha-1, \alpha+1]}+\Theta_{\alpha+1}^{[p-\alpha-1, \alpha+1]}=0$ with both summands non-zero, while for the remaining $\Theta_{\alpha}^{[p-\alpha, \alpha]}=\Theta_{\alpha}^{[p-\alpha+1, \alpha-1]}=\Theta_{\alpha+2}^{[p-\alpha-2, \alpha+3]}=$ $\Theta_{\alpha+1}^{[p-\alpha-2, \alpha+2]}=0$.

(3) If $\beta=\alpha+3$, then

$$
\left[X, \Theta^{[p-\beta, \beta]}\right]=\Theta_{\alpha+3}^{[p-\alpha-4, \alpha+4]}+\Theta_{\alpha+3}^{[p-\alpha-3, \alpha+3]}+\Theta_{\alpha+3}^{[p-\alpha-2, \alpha+2]},
$$

and it follows at once that $\left[X, \Theta^{[p-\alpha, \alpha]}\right]=\left[X, \Theta^{[p-\beta, \beta]}\right]=0$, which is excluded by assumption. The same result is obtained for indices $\beta=\alpha+k$ for $k \geqslant 4$. 
This shows that if a Casimir operator of $\mathfrak{s}$ decomposes like $(21)$, then necessarily $1 \leqslant \mid \beta-$ $\alpha \mid \leqslant 2$. Note that this argument trivially extends to the case $C_{p}=\Theta^{[p, 0]}+\Theta^{[p-\alpha, \alpha]}+\Theta^{[p-\beta, \beta]}$, since $\Theta^{[p, 0]}$, as an invariant of the subalgebra $\mathfrak{s}^{\prime}$, has no effect on the scalars. In particular, for the quadratic Casimir operators we will always obtain the decomposition $C_{2}=\Theta^{[2,0]}+\Theta^{[0,2]}$.

The same ansatz for decompositions with more components enables us to establish the following.

Criterion A. If $C_{p}$ decomposes as $C_{p}=\lambda \Theta^{[p, 0]}+\Theta^{[p-\alpha, \alpha]}+\Theta^{[p-\beta, \beta]}+\Theta^{[p-\beta-2, \beta+2]}$ with $|\beta-\alpha| \leqslant 2$ and $\lambda=0,1$, then

$\left[\Theta^{[p-\alpha, \alpha]}, \Theta^{[p-\beta, \beta]}\right]=\left[\Theta^{[p-\alpha, \alpha]}, \Theta^{[p-\beta-2, \beta+2]}\right]=\left[\Theta^{[p-\beta, \beta]}, \Theta^{[p-\beta-2, \beta+2]}\right]=0$.

Clearly for any of the scalars we immediately obtain $\left[C_{p}, \Theta^{[p-\mu, \mu]}\right]=0(\mu=\alpha, \beta, \beta+2)$. The single commutators are given by formula (17):

$$
\begin{aligned}
& {\left[\Theta^{[p-\alpha, \alpha]}, \Theta^{[p-\beta, \beta]}\right]=\Theta^{[2 p-\alpha-\beta-1, \alpha+\beta]}+\Theta^{[2 p-\alpha-\beta, \alpha+\beta-1]}+\Theta^{[2 p-\alpha-\beta+1, \alpha+\beta-2]},} \\
& {\left[\Theta^{[p-\alpha, \alpha]}, \Theta^{[p-\beta-2, \beta+2]}\right]=\Theta^{[2 p-\alpha-\beta-3, \alpha+\beta+2]}+\Theta^{[2 p-\alpha-\beta-2, \alpha+\beta+1]}+\Theta^{[2 p-\alpha-\beta-1, \alpha+\beta]},} \\
& {\left[\Theta^{[p-\beta, \beta]}, \Theta^{[p-\beta-2, \beta+2]}\right]=\Theta^{[2 p-2 \beta-3,2 \beta+2]}+\Theta^{[2 p-2 \beta-2,2 \beta+1]}+\Theta^{[2 p-2 \beta-1,2 \beta]} .}
\end{aligned}
$$

The commutator $\left[C_{p}, \Theta^{[p-\beta, \beta]}\right]=0$ implies that the operators on the right-hand side of (24a) and (24c) must sum to zero. Comparing the bi-degrees of these operators, it follows immediately that

$$
\left[\Theta^{[p-\alpha, \alpha]}, \Theta^{[p-\beta, \beta]}\right]=\left[\Theta^{[p-\beta, \beta]}, \Theta^{[p-\beta-2, \beta+2]}\right]=0 .
$$

Now evaluating $\left[C_{p}, \Theta^{[p-\alpha, \alpha]}\right]=0$ and taking into account (25), we obtain the identity $\left[\Theta^{[p-\alpha, \alpha]}, \Theta^{[p-\beta-2, \beta+2]}\right]=0$, proving that the subgroup scalars mutually commute. Observe that again the presence of $\Theta^{[p, 0]}$ in the decomposition has no effect.

Using this property, we can refine it to a criterion for the commutativity of subgroup scalars of different total degree, which is proved in a completely analogous way.

Criterion B. Let $C_{p}=\Theta^{[p-\alpha, \alpha]}+\Theta^{[p-\beta, \beta]}+\Theta^{[p-\gamma, \gamma]}(0 \neq \alpha<\beta<\gamma)$ be a Casimir operator of $\mathfrak{s}$ with $\gamma-\alpha \geqslant 3$. If $\Theta^{[r, s]}$ is a subgroup scalar of $\mathfrak{s}^{\prime} \subset \mathfrak{s}$ such that $\left[\Theta^{[r, s]}, \Theta^{[p-\beta, \beta]}\right]=0$, then $\left[\Theta^{[r, s]}, \Theta^{[p-\alpha, \alpha]}\right]=0$ and $\left[\Theta^{[r, s]}, \Theta^{[p-\gamma, \gamma]}\right]=0$.

For four or more scalars in the decomposition of $C_{p}$, the terms do no more necessarily commute with each other. However, for decomposition of the form $C_{p}=\Theta^{[p, 0]}+\Theta^{\left[p-\alpha_{1}, \alpha_{1}\right]}+$ $\Theta^{\left[p-\alpha_{2}, \alpha_{2}\right]}+\ldots+\Theta^{\left[p-a_{k}, a_{k}\right]}+\Theta^{\left[p-\alpha_{k}-2, \alpha_{k}+2\right]}$, it is still true that $\left[\Theta^{\left[p-a_{k}, a_{k}\right]}, \Theta^{\left[p-\alpha_{k}-2, \alpha_{k}+2\right]}\right]=0$. Moreover, both criteria $\mathrm{A}$ and $\mathrm{B}$ can be expressed in an analytical way, replacing the commutator by the Berezin bracket of the projected scalars $\pi\left(\Theta^{[p, q]}\right):=O^{[p, q]}$.

\section{Algorithm}

Based on the preceding results on the decomposition of Casimir operators, the Berezin bracket and criteria $\mathrm{A}$ and $\mathrm{B}$, we can propose an analytical procedure to determine a set of missing label operators. This procedure will be applicable to reduction chains $\mathfrak{s} \supset \mathfrak{s}^{\prime}$ of semisimple Lie algebras, the MLP of which has $n$-labelling operators.

The algorithm is developed in the analytical frame, i.e. considering the analytical counterpart of Casimir operators and subgroup scalars. Following the notations of previous sections, we will denote by $\pi\left(C_{p}\right)$ the projection of Casimir operators (also called Casimir invariants) and by $O^{[p-\alpha, \alpha]}$ the projected subgroup scalars $\pi\left(\Theta^{[p-\alpha, \alpha]}\right)$. Criteria A and B are also assumed in its analytical version.

5 For this reason, we usually leave out the quadratic Casimir operators. 
(i) Decompose the Casimir invariants of $\mathfrak{s}$ of degree $p \geqslant 3$ using the scaling transformation (10): $\pi\left(C_{p}\right)=\sum_{\alpha=0}^{M_{p}} \pi\left(\Theta^{[p-\alpha, \alpha]}\right)=\sum_{\alpha=0}^{M_{p}} O^{[p-\alpha, \alpha]}$.

(ii) Determine if there are scalars satisfying the criterion $\mathrm{A}$.

(iii) For the non-factorizable pairs $\left(O^{[p-\alpha, \alpha]}, O^{[q-\alpha, \alpha]}\right)$ not satisfying the criterion A compute the Berezin bracket.

(iv) Determine if there are scalars satisfying the criterion B.

(v) Let $t$ be the total number of mutually involutive operators $O^{[p-\alpha, \alpha]}$.

(a) If $t<n$, proceed to step (vi).

(b) If $t \geqslant n$ mutually involutive operators $O^{[p-\alpha, \alpha]}$ are found, compute the rank of the system $\mathcal{L}=\left\{\pi\left(C_{1}\right), \ldots, \pi\left(C_{l}\right), \pi\left(C_{1}^{\prime}\right), \ldots, \pi\left(C_{l^{\prime}}^{\prime}\right), O^{\left[p_{1}-\alpha_{1}, \alpha_{1}\right]}, \ldots, O^{\left[p_{t}-\alpha_{t}, \alpha_{t}\right]}\right\}$.

(1) If $\operatorname{rank}(\mathcal{L}) \geqslant l+l^{\prime}-l_{0}+n$, the symmetrized representatives of the operators solve the missing labelling problem: $\left[l=\operatorname{rank}(\mathfrak{s}), l^{\prime}=\operatorname{rank}\left(\mathfrak{s}^{\prime}\right)\right]$.

(2) If $\operatorname{rank}(\mathcal{L})<l+l^{\prime}-l_{0}+n$, proceed to step (vi). (The MLP is not solved with decomposition only.)

(vi) Determine further $t^{\prime} \geqslant n-\operatorname{rank}(\mathcal{L})$ subgroup scalars $\Phi_{1}, \ldots, \Phi_{t^{\prime}}$ such that

(a) $\left\{\Phi_{k}, O^{\left[p_{i}-\alpha_{i}, \alpha_{i}\right]}\right\}=0$ for $k=1, \ldots, t^{\prime}$ and $i=1, \ldots, t$;

(b) $\left\{\Phi_{k}, \Phi_{l}\right\}=0$ for $k, l=1, \ldots, t^{\prime}$;

(c) $\operatorname{rank}\left(\left\{\pi\left(C_{1}\right), \ldots, \pi\left(C_{l}\right), \pi\left(C_{1}^{\prime}\right), \ldots, \pi\left(C_{l^{\prime}}^{\prime}\right), O^{\left[p_{1}-\alpha_{1}, \alpha_{1}\right]}, \ldots, O^{\left[p_{t}-\alpha_{t}, \alpha_{t}\right]}, \Phi_{1}, \ldots\right.\right.$, $\left.\left.\Phi_{t^{\prime}}\right\}\right) \geqslant n$.

Extract $n$ functionally independent operators from the previous system and take their symmetric representatives.

The sixth step of the algorithm is the less automatic one, and certainly one of difficult execution, in either the analytical or algebraic way. Either solving the systems of partial differential equations for these scalars or trying to establish the corresponding generating functions [2] is a laborious task, the difficulty of which increases rapidly for higher rank algebras. Since this step cannot be described in terms of the invariants of the intervening algebras, a further systematization is not possible.

\subsection{Orthonormal bases of eigenstates}

To completely describe the states of IRs of $\mathfrak{s}$, besides the Casimir operators of $\mathfrak{s}$ and $\mathfrak{s}^{\prime}$ and the labelling operators, we will also need some appropriate internal subgroup labels, as follows from formula (1). The missing labelling operators are subgroup scalars; thus, they will automatically commute with these internal subgroup operators, as the latter depend only on the subgroup generators ${ }^{6}$. In general, the missing label operators will separate the degeneracies occurring whenever two or more copies of an IR of $\mathfrak{s}^{\prime}$ appear when reducing an IR of $\mathfrak{s}$. The internal labels, in contrast, will distinguish the different eigenvectors within each irreducible representation of $\mathfrak{s}^{\prime}$.

In this section we outline a possible generic procedure to construct an orthonormal basis of states for a given irreducible representation $R$ of $\mathfrak{s}$. In the following we assume that $\mathfrak{s}$ and $\mathfrak{s}^{\prime}$ are semisimple Lie algebras, and that the operators constructed in the enveloping algebra are Hermitian.

We fix the following notations:

- $C_{1}, \ldots, C_{l}$-Casimir operators of $\mathfrak{s}$,

- $C_{1}^{\prime}, \ldots, C_{l^{\prime}}^{\prime}$ - Casimir operators of $\mathfrak{s}^{\prime}$,

${ }^{6}$ Typically these internal labels are associated with the Casimir operators of suitable chosen subalgebras of $\mathfrak{s}^{\prime}$ or generators of the Cartan subalgebra. 
- $\Theta_{1}, \ldots, \Theta_{n}$ - the missing label operators,

- $J_{1}, \ldots, J_{f}$-internal subgroup operators.

In order to sum up the required number of labels (1), the quantity of internal subgroup labels must be $f=\frac{1}{2}\left(\operatorname{dim} \mathfrak{s}-l-2 l^{\prime}-2 n+2 l_{0}\right)=\frac{1}{2}\left(\operatorname{dim} \mathfrak{s}^{\prime}-l^{\prime}\right)$.

Let $R=\left[\mu_{1}, \ldots, \mu_{l}\right]$ be a given irreducible representation of $\mathfrak{s}$. For any Casimir operator $C_{k}$ we have

$$
\left\langle\mu_{1}, \ldots, \mu_{l}\left|C_{k}\right| \mu_{1}, \ldots, \mu_{l}\right\rangle=\left\langle C_{k}\right\rangle, k=1, \ldots, l .
$$

The eigenvalues $\left\langle C_{1}\right\rangle, \ldots,\left\langle C_{l}\right\rangle$ therefore characterize $R$ and will be the same for any particular state within the representation. For this reason, and whenever there is no ambiguity concerning $R$, we may skip these eigenvalues.

In our construction of an orthonormal basis of $R$, the first step is to consider the branching rules of the chain $\mathfrak{s} \supset \mathfrak{s}^{\prime}$, in order to obtain the decomposition of $R$ into a sum of IRs of the subalgebra $\mathfrak{s}^{\prime}$ :

$$
R=R_{1}^{m_{1}} \oplus \cdots \oplus R_{q}^{m_{q}} .
$$

For each $i \neq j$ we assume that $R_{i} \neq R_{j}$, i.e. they are non-equivalent irreducible representations. The superindex $m_{i} \geqslant 1(i=1, \ldots, q)$ denotes the multiplicity of $R_{i}$ in $R$. Further let $d_{i}=\operatorname{dim} R_{i}$. In view of this decomposition, we can determine a basis $\mathfrak{B}=\left\{\left|\psi_{i}^{j}\right\rangle, i=1, \ldots, d_{i} m_{i} ; j=1, \ldots, q\right\}$ of the representation space of $R$ such that for fixed $j_{0}$, the vectors $\left|\psi_{i}^{j_{0}}\right\rangle\left(i=1, \ldots, d_{j_{0}} m_{j_{0}}\right)$ form a basis of $R_{j_{0}}^{m_{j_{0}}}$. This can be done starting from an arbitrary nonzero vector in $R$ and analysing how it transforms by the generators of $\mathfrak{s}^{\prime}$ (once an IR $R^{\prime}$ of the latter algebra has been recovered, the process is repeated for another vector not lying in $R^{\prime}$, and so on, up to covering the whole space of $R$ ). This procedure moreover allows us to separate the copies of $R_{j_{0}}$, so that we can suppose that $\mathfrak{B}$ is arranged in such manner that for $\alpha=0, \ldots, m_{j_{0}}-1$, the vectors

$$
\left|\psi_{\alpha d_{j_{0}}+i}^{j_{0}}\right\rangle, \quad\left(i=1, \cdots, d_{j_{0}}\right)
$$

are a basis of the $(\alpha+1)$ st copy of $R_{j_{0}}$. Now any Casimir operator $C_{r}^{\prime}\left(r=1, \ldots, l^{\prime}\right)$ of $\mathfrak{s}^{\prime}$ is diagonal over the irreducible representation $R_{i}$, with the corresponding eigenvalue $\lambda_{i}^{r}$; hence, we get that for the (reducible) representation $R$ the operator $C_{r}^{\prime}$ is given by the block matrix ${ }^{7}$

$$
C_{r}^{\prime}(R)=\left(\begin{array}{ccc}
\lambda_{1}^{r} \operatorname{Id}_{d_{1} m_{1}} & & \\
& \ddots & \\
& & \lambda_{q}^{r} \operatorname{Id}_{d_{q} m_{q}}
\end{array}\right) \quad\left(r=1, \cdots, l^{\prime}\right),
$$

where $\operatorname{Id}_{d_{a} m_{a}}$ denotes the $\left(d_{a} m_{a}\right)$-dimensional unit matrix for $a=1, \ldots, q$.

The second step is to adequately modify the basis (28) to obtain a basis of eigenvectors for the labelling operators $\Theta_{1}, \ldots, \Theta_{n}$. These commute with the Casimir operators $C_{1}^{\prime}, \ldots, C_{l^{\prime}}^{\prime}$ of $\mathfrak{s}^{\prime}$; thus, for any fixed index $j_{0}=1, \ldots, q$ and vector $\left|\psi_{i}^{j_{0}}\right\rangle$ we get the relation

$$
C_{r}^{\prime} \Theta_{k}\left|\psi_{i}^{j_{0}}\right\rangle=\Theta_{k} C_{r}^{\prime}\left|\psi_{i}^{j_{0}}\right\rangle=\lambda_{i}^{r} \Theta_{k}\left|\psi_{i}^{j_{0}}\right\rangle
$$

which means that $\Theta_{k}\left|\psi_{i}^{j_{0}}\right\rangle$ has the same eigenvalues for the Casimir operators of $\mathfrak{s}^{\prime}$ as the vector $\left|\psi_{i}^{j_{0}}\right\rangle$. Therefore,

$$
\Theta_{k}\left|\psi_{i}^{j_{0}}\right\rangle=\sum_{p=1}^{d_{j_{0}} m_{j_{0}}} \alpha_{j_{0} k}^{p}\left|\psi_{p}^{j_{0}}\right\rangle
$$

7 To denote the matrix of an operator $T$ on a particular representation $R$, we will use the symbol $T(R)$. 
for some coefficients $\alpha_{j_{0} k}^{p}$. In terms of matrices (see (29)), the preceding equation means that $\Theta_{k}(R)$ has the following block matrix structure:

$$
\Theta_{k}(R)=\left(\begin{array}{ccc}
\mathrm{A}_{1}^{k} & 0 & 0 \\
0 & \ddots & 0 \\
0 & 0 & \mathrm{~A}_{q}^{k}
\end{array}\right)
$$

where each $\mathrm{A}_{i}^{k}$ is an $\left(m_{i} d_{i} \times m_{i} d_{i}\right)$ matrix. These blocks $\mathrm{A}_{i}^{k}$ need not to be diagonal themselves, but since the $\Theta_{k}$ are Hermitian operators for each $j_{0}=1, \ldots, q$ we can always transform the basis $\left|\psi_{i}^{j_{0}}\right\rangle$ to a basis $\left|\widehat{\psi}_{i}^{j_{0}}\right\rangle$ of $R_{j_{0}}^{m_{j_{0}}}$ such that

$$
\Theta_{k}\left|\widehat{\psi}_{\alpha d_{j_{0}}+i}^{j_{0}}\right\rangle=\xi_{k, \alpha}^{j_{0}}\left|\widehat{\psi}_{\alpha d_{j_{0}}+i}^{j_{0}}\right\rangle
$$

for $i=1, \ldots, d_{j_{0}}$ and $\alpha=0, \ldots, m_{j_{0}}-1$. Over this new basis of $R_{j_{0}}^{m_{j_{0}}}$, the matrix of $\Theta_{k}$ (restricted to the latter subspace) is given by its eigenvalues on the different copies of $R_{j_{0}}$ :

$$
\Theta_{k}\left(R_{j_{0}}^{m_{j_{0}}}\right)=\left(\begin{array}{ccc}
\xi_{k, 1}^{j_{0}} \mathrm{Id}_{d_{j_{0}}} & 0 & 0 \\
0 & \ddots & 0 \\
0 & 0 & \xi_{k, m_{j_{0}}}^{j_{0}} \mathrm{Id}_{d_{j_{0}}}
\end{array}\right)
$$

Up to this step, we have separated the different irreducible representations of $\mathfrak{s}^{\prime}$ and their multiplicities by the eigenvalues of the Casimir operators of $\mathfrak{s}^{\prime}$ and the labelling operators. It now remains to separate the states within each irreducible IR. This is done diagonalizing the matrices $J_{p}(R)$ of the internal subgroup operators $J_{p}(p=1, \ldots, f)$. Because of the relation

$$
J_{p} C_{r}^{\prime}\left|\widehat{\psi}_{\alpha d_{j_{0}}+i}^{j_{0}}\right\rangle=\lambda_{i}^{r}\left(J_{p}\left|\widehat{\psi}_{\alpha d_{j_{0}}+i}^{j_{0}}\right\rangle\right)=C_{r}^{\prime} J_{p}\left|\widehat{\psi}_{\alpha d_{j_{0}}+i}^{j_{0}}\right\rangle
$$

it suffices again to see what happens for the restrictions of $R$ to the (reducible) representations $R_{i}^{m_{i}}$. By (33), we further have that

$$
J_{p} \Theta_{k}\left|\widehat{\psi}_{\alpha d_{j_{0}}+i}^{j_{0}}\right\rangle=\xi_{k, \alpha}^{j_{0}}\left(J_{p}\left|\widehat{\psi}_{\alpha d_{j_{0}}+i}^{j_{0}}\right\rangle\right)=\Theta_{k} J_{p}\left|\widehat{\psi}_{\alpha d_{j_{0}}+i}^{j_{0}}\right\rangle
$$

which implies that the vector $J_{p}\left|\widehat{\psi}_{\alpha d_{j_{0}}+i}^{j_{0}}\right\rangle$ also has the same eigenvalues for each $\Theta_{k}$ than $\left|\widehat{\psi}_{\alpha d_{j_{0}}+i}^{j_{0}}\right\rangle$. As the $J_{p}$ are diagonalizable operators, for each $j_{0}=1, \ldots, q$ and $\alpha=0, \ldots, m_{j_{0}}-1$ we can find adequate linear combinations

$$
\left|\widetilde{\psi}_{\alpha d_{j_{0}}+i}^{j_{0}}\right\rangle=\sum \beta_{j_{0} \alpha}^{t}\left|\widehat{\psi}_{\alpha d_{j_{0}}+t}^{j_{0}}\right\rangle
$$

such that the condition $J_{p}\left|\widetilde{\psi}_{\alpha d_{j_{0}}+i}^{j_{0}}\right\rangle=\varphi_{i}^{p}\left|\widetilde{\psi}_{\alpha d_{j_{0}}+i}^{j_{0}}\right\rangle$ holds.

In this final basis $\mathfrak{B}^{\prime}=\left\{\left|\widetilde{\psi}_{\alpha d_{j}+i}^{j}\right\rangle ; i=1, \ldots, d_{j} ; \alpha=0, \ldots, m_{j}-1 ; j=1, \ldots, q\right\}$, each vector is characterized by its eigenvalues

$$
\left|\widetilde{\psi}_{\alpha d_{j}+i}^{j}\right\rangle=\left|\lambda_{1}, \ldots, \lambda_{l^{\prime}} ; \xi_{1}, \ldots, \xi_{n} ; \varphi_{1}, \ldots, \varphi_{f}\right\rangle
$$

for $C_{1}^{\prime}, \ldots, C_{l^{\prime}}^{\prime}, \Theta_{1}, \ldots, \Theta_{n}, J_{1}, \ldots, J_{f}$ (we may add the eigenvalues (26) of the Casimir operators of $\mathfrak{s}$ in the case of ambiguity).

The basis $\mathfrak{B}^{\prime}$ is orthogonal by construction. As $\left|\widetilde{\psi}_{\alpha d_{j_{0}}+i}^{j_{0}}\right\rangle$ is characterized by its eigenvalues for any arbitrary pair of distinct vectors $\left|\widetilde{\psi}_{\alpha_{0} d_{j_{0}}+i_{0}}^{j_{0}}\right\rangle$ and $\left|\widetilde{\psi}_{\alpha_{1} d_{j_{1}}+i_{1}}^{j_{1}}\right\rangle$ there is at least one operator $T$ among $C_{1}^{\prime}, \ldots, C_{l^{\prime}}^{\prime}, \Theta_{1}, \ldots, \Theta_{n}, J_{1}, \ldots, J_{f}$ such that $T\left|\widetilde{\psi}_{\alpha_{0} d_{j_{0}}+i_{0}}^{j_{0}}\right\rangle=$ 
$\chi_{0}\left|\widetilde{\psi}_{\alpha_{0} d_{j_{0}}+i_{0}}^{j_{0}}\right\rangle, T\left|\widetilde{\psi}_{\alpha_{1} d_{j_{1}}+i_{1}}^{j_{1}}\right\rangle=\chi_{1}\left|\widetilde{\psi}_{\alpha_{1} d_{j_{1}}+i_{1}}^{j_{1}}\right\rangle$ with $\chi_{0} \neq \chi_{1}$. Since $T$ is Hermitian ${ }^{8}$, we obtain that

$$
\begin{gathered}
\left\langle\widetilde{\psi}_{\alpha_{0} d_{j_{0}}+i_{0}}^{j_{0}}|T| \widetilde{\psi}_{\alpha_{1} d_{j_{1}}+i_{1}}^{j_{1}}\right\rangle=\left\langle\widetilde{\psi}_{\alpha_{0} d_{j_{0}+i_{0}}}^{j_{0}} \chi_{1} \mid \widetilde{\psi}_{\alpha_{1} d_{j_{1}}+i_{1}}^{j_{1}}\right\rangle=\chi_{1}\left\langle\widetilde{\psi}_{\alpha_{0} d_{j_{0}}+i_{0}}^{j_{j_{0}}} \mid \widetilde{\psi}_{\alpha_{1} d_{j_{1}}+i_{1}}^{j_{1}}\right\rangle, \\
\left.\times\left\langle\widetilde{\psi}_{\alpha_{0} d_{j_{0}}+i_{0}}^{j_{0}} \mid \chi_{0} \widetilde{\psi}_{\alpha_{1}}^{j_{1} d_{j_{1}}+i_{1}}\right\rangle=\chi_{0}\left|\widetilde{\psi}_{\alpha_{0} d_{j_{0}}+i_{0}}^{j_{0}}\right| \widetilde{\psi}_{\alpha_{1} d_{j_{1}}+i_{1}}^{j_{1}}\right\rangle,
\end{gathered}
$$

hence that $\left(\chi_{1}-\chi_{0}\right)\left\langle\widetilde{\psi}_{\alpha_{0} d_{j_{0}}+i_{0}}^{j_{0}} \mid \widetilde{\psi}_{\alpha_{1} d_{j_{1}}+i_{1}}^{j_{1}}\right\rangle=0$, from which the orthogonality follows. The last step, the orthonormalization of the basis $\mathfrak{B}^{\prime}$, is a routine computation that follows from the application of the Gram-Schmidt method.

The practical receipt to diagonalize the operators can be roughly summarized in the following five steps.

(i) Decompose the IR $R$ of $\mathfrak{s}$ into IRs of $\mathfrak{s}^{\prime}: R=R_{1}^{m_{1}} \oplus \cdots \oplus R_{q}^{m_{q}}$.

(ii) For each $R_{i}^{m_{i}}(i=1, \ldots, q)$ determine a basis of eigenvectors for the Casimir operators of $\mathfrak{s}^{\prime}$.

(iii) Using (30), find a basis of $R_{i}^{m_{i}}$ that diagonalizes the labelling operators $\Theta_{k}(k=1, \ldots, n)$.

(iv) Within any IR $R_{i}$, diagonalize the internal subgroup operators $J_{1}, \ldots, J_{f}$.

(v) Apply Gram-Schmidt to orthonormalize the different eigenspaces.

It should be noted that this is only one among the various different possibilities to construct orthonormal bases. Often valuable information concerning an orthonormal basis, like its spectrum, can be more conveniently derived by comparison with another (analytical) basis. For example, canonical bases like the Gel'fand-Tseitlin patterns are suitable for labelling problems associated with unitary groups $[10,12]$.

\section{Examples}

To illustrate the implementation of the algorithm and the construction of orthonormal bases, we develop in this section four labelling problems with $n=1,3,6$ and $n=3$ missing labels, respectively. For the first example we diagonalize the missing label operator and give the complete basis of eigenstates for the lowest dimensional IR exhibiting multiplicities when reduced to a subalgebra. The second and third examples (with $n=3$ and $n=6$ missing labels, respectively) can be solved using steps (i)-(v) of the algorithm. The last example presents a situation where step (vi) is required to obtain the adequate number of missing labels. We remark that some of these examples have been analysed in the literature from alternative or different points of view $[2,5,7,9,13]$.

\subsection{The chain $G_{2} \supset \mathfrak{s u}(3)$}

As a first example we analyse the reduction $G_{2} \supset \mathfrak{s u}(3)$ with $n=1$ missing label for generic irreducible representations [2, 9]. We consider the same basis considered in [14], consisting of the generators $g_{k}^{l}, a_{k}^{+}, a_{k}^{-}=\left(a_{k}^{+}\right)^{\dagger}$ with indices $1 \leqslant k, l \leqslant 3$ and the constraints $\left(g_{k}^{l}\right)^{\dagger}=g_{l}^{k}$, $\sum_{k=1}^{3} g_{k}^{k}=0$. The commutation relations of $G_{2}$ are given by

$$
\begin{aligned}
& {\left[g_{k}^{l}, g_{m}^{n}\right]=\delta_{m}^{l} g_{k}^{n}-\delta_{k}^{n} g_{m}^{l}, \quad\left[g_{k}^{l}, a_{m}^{ \pm}\right]= \pm \delta_{m}^{l} a_{k}^{ \pm} \mp \frac{1}{3} \delta_{k}^{l} a_{m}^{ \pm},} \\
& {\left[a_{k}^{+}, a_{m}^{-}\right]=g_{k}^{m}, \quad\left[a_{k}^{ \pm}, a_{l}^{ \pm}\right]=\mp \frac{2}{\sqrt{3}} \varepsilon_{k l m} a_{m}^{\mp} .}
\end{aligned}
$$

8 Actually, the orthogonality of eigenvectors corresponding to different vectors holds for a more general type of operators, the so-called normal operators $\left(T T^{\dagger}=T^{\dagger} T\right)$. 
Clearly, the $g_{k}^{l}$ span the $\mathfrak{s u ( 3 )}$ subalgebra. Using the expression derived in [14] for the sixth-order Casimir operator of $G_{2}$, we obtain that it decomposes as the sum of the following subgroup scalars:

$$
C_{6}=\Theta^{[6,0]}+\Theta^{[4,2]}+\Theta^{[3,3]}+\Theta^{[2,4]}+\Theta^{[0,6]} \text {. }
$$

It can be easily shown that $\Theta^{[0,6]}=\frac{11}{144}\left(\Theta^{[0,2]}\right)^{3}$; thus $\Theta^{[0,6]}$ will not provide an independent operator. Hence we may take $\Theta^{[2,4]}$ as the missing label operator. A routine computation shows that $C_{2}, C_{6}, C_{2}^{\prime}, C_{3}^{\prime}, \Theta^{[2,4]}$ are independent. As internal $\mathfrak{s u}(3)$ operators we consider the generators $g_{1}^{1}, g_{2}^{2}$ and $\left(g_{1}^{1}\right)^{2}+\left(g_{2}^{2}\right)^{2}+2\left(g_{1}^{2} g_{2}^{1}+g_{2}^{1} g_{1}^{2}-g_{1}^{1} g_{2}^{2}\right)$, the latter being the Casimir operator of the (canonical) $\mathfrak{s o}(3)$ subalgebra of $\mathfrak{s u}(3)$ generated by $g_{1}^{2}, g_{2}^{1}$ and $g_{1}^{1}-g_{2}^{2}$. It is straightforward to verify that all these operators are Hermitian and commute, and thus that they are simultaneously diagonalizable. An orthonormal basis, constructed along the lines described in the previous section, will be given by the eigenvectors $\left|\lambda_{1}, \lambda_{2} ; \xi ; \varphi_{1}, \varphi_{2}, \varphi_{3}\right\rangle$, where $\lambda_{i}$ denote the eigenvalues of the $\mathfrak{s u}(3)$ Casimir operators, $\xi$ that of $\Theta^{[2,4]}$ and the $\varphi_{i}$ 's those of the internal labels.

In table 1 we give the corresponding eigenvalues for the 64 states of the IR $R=[11]$. When reduced with respect to $\mathfrak{s u}(3)$, this representation decomposes as

$$
[11] \supset(12)+(21)+(11)+(11)+(02)+(20)+(01)+(10)
$$

We observe that the adjoint representation (11) of $\mathfrak{s u ( 3 )}$ appears twice; thus, the missing label is required to separate both copies.

\subsection{The chain $\mathfrak{s o}(7) \supset \mathfrak{s u}(2)^{3}$}

The missing label problem associated with these Lie algebras has $n=3$ missing labels for generic representations. A pair of commuting scalars was found in [6] using a procedure specially developed for labelling problems involving $[\mathfrak{s u}(2)]^{p}$-subalgebras, which however does not provide the third required commuting operator.

We consider the basis of $\mathfrak{s o}(7)$ taken in [6, 13], consisting of generators $S_{0, \pm 1}, U_{0, \pm 1}, W_{0, \pm 1}$ that span the subalgebra $\mathfrak{s u}(2)^{3}$, together with a tensor operator $T_{\lambda, \mu, \nu}^{1, \frac{1}{2}, \frac{1}{2}}$. The latter has the property that its commutators only produce generators of the subalgebra [13].

Over this basis, the Casimir operators of $\mathfrak{s o}(7)$ can be easily constructed by the usual trace method. Consider the matrix $M$ defined by

$$
\left[\begin{array}{ccccccc}
\sqrt{2}\left(u_{0}+w_{0}\right) & -2 w_{-1} & 2 t_{1,-\frac{1}{2},-\frac{1}{2}} & 2 t_{0,-\frac{1}{2},-\frac{1}{2}} & 2 t_{-1,-\frac{1}{2},-\frac{1}{2}} & 2 u_{-1} & 0 \\
-2 w_{1} & \sqrt{2}\left(u_{0}-w_{0}\right) & 2 t_{1,-\frac{1}{2}, \frac{1}{2}} & 2 t_{0,-\frac{1}{2}, 1 / 2} & 2 t_{-1,-\frac{1}{2}, \frac{1}{2}} & 0 & 2 u_{-1} \\
2 t_{-1,1 / 2, \frac{1}{2}} & -2 t_{-1, \frac{1}{2},-\frac{1}{2}} & \sqrt{2} s_{0} & \sqrt{2} s_{-1} & 0 & 2 t_{-1,-\frac{1}{2}, \frac{1}{2}} & -2 t_{-1,-\frac{1}{2},-\frac{1}{2}} \\
-2 t_{0, \frac{1}{2}, \frac{1}{2}} & 2 t_{0, \frac{1}{2},-\frac{1}{2}} & -\sqrt{2} s_{1} & 0 & \sqrt{2} s_{-1} & -2 t_{0,-\frac{1}{2}, \frac{1}{2}} & 2 t_{0,-\frac{1}{2},-\frac{1}{2}} \\
2 t_{1,1 / 2, \frac{1}{2}} & -2 t_{1, \frac{1}{2},-\frac{1}{2}} & 0 & -\sqrt{2} s_{1} & -\sqrt{2} s_{0} & 2 t_{1,-\frac{1}{2}, \frac{1}{2}} & -2 t_{1,-\frac{1}{2},-\frac{1}{2}} \\
-2 u_{1} & 0 & -2 t_{1, \frac{1}{2},-\frac{1}{2}} & -2 t_{0, \frac{1}{2},-\frac{1}{2}} & -2 t_{-1, \frac{1}{2},-\frac{1}{2}}-\sqrt{2}\left(u_{0}-w_{0}\right) & -2 w_{-1} \\
0 & -2 u_{1} & -2 t_{1, \frac{1}{2}, \frac{1}{2}} & -2 t_{0, \frac{1}{2}, 1 / 2} & -2 t_{-1, \frac{1}{2}, \frac{1}{2}} & -2 w_{1} & -\sqrt{2}\left(u_{0}+w_{0}\right)
\end{array}\right] .
$$

The matrix $M$ satisfies the conditions $\operatorname{Tr}(M)=\operatorname{Tr}\left(M^{3}\right)=\operatorname{Tr}\left(M^{5}\right)=\operatorname{Tr}\left(M^{7}\right)=0$ and $\operatorname{Tr}\left(M^{2 k}\right)=\Phi_{2 k}\left(s_{\alpha}, u_{\alpha}, w_{\alpha}, t_{\lambda, \mu, \nu}\right)(k=1,2,3)$. The symmetrized polynomials 
Table 1. Basis of eigenstates $\left|\lambda_{1}, \lambda_{2} ; \xi ; \varphi_{1}, \varphi_{2}, \varphi_{3}\right\rangle$ for $G_{2} \supset \mathfrak{s u}(3)$ and $R=[11]$.

\begin{tabular}{|c|c|c|c|c|c|c|c|c|c|c|c|}
\hline$\lambda_{1}$ & $\lambda_{2}$ & $\xi$ & $\varphi_{1}$ & $\varphi_{2}$ & $\varphi_{3}$ & $\lambda_{1}$ & $\lambda_{2}$ & $\xi$ & $\varphi_{1}$ & $\varphi_{2}$ & $\varphi_{3}$ \\
\hline 16 & -56 & $\frac{126289}{2}$ & -2 & -1 & $\frac{11}{2}$ & 9 & 0 & $\frac{667239}{4}$ & -2 & 1 & $\frac{11}{2}$ \\
\hline 16 & -56 & $\frac{126289}{2}$ & 0 & 1 & $\frac{3}{2}$ & 9 & 0 & $\frac{667239}{4}$ & 2 & -1 & $\frac{11}{2}$ \\
\hline 16 & -56 & $\frac{126289}{2}$ & -3 & 1 & $\frac{21}{2}$ & 9 & 0 & $\frac{667239}{4}$ & 1 & -2 & 4 \\
\hline 16 & -56 & $\frac{126289}{2}$ & 1 & -1 & $\frac{5}{2}$ & 9 & 0 & $\frac{667239}{4}$ & -1 & 2 & 4 \\
\hline 16 & -56 & $\frac{126289}{2}$ & 0 & -2 & 3 & 9 & 0 & $\frac{667239}{4}$ & 0 & 0 & 0 \\
\hline 16 & -56 & $\frac{126289}{2}$ & -2 & 2 & 7 & 9 & 0 & $\frac{667239}{4}$ & 0 & 0 & 6 \\
\hline 16 & -56 & $\frac{126289}{2}$ & -1 & 0 & 7 & 9 & 0 & $\frac{417759}{4}$ & -1 & -1 & $\frac{5}{2}$ \\
\hline 16 & -56 & $\frac{126289}{2}$ & -1 & 0 & 1 & 9 & 0 & $\frac{417759}{4}$ & 1 & 1 & $\frac{5}{2}$ \\
\hline 16 & -56 & $\frac{126289}{2}$ & 1 & 2 & 4 & 9 & 0 & $\frac{417759}{4}$ & -2 & 1 & $\frac{11}{2}$ \\
\hline 16 & -56 & $\frac{126289}{2}$ & 1 & -1 & $\frac{23}{2}$ & 9 & 0 & $\frac{417759}{4}$ & 2 & -1 & $\frac{11}{2}$ \\
\hline 16 & -56 & $\frac{126289}{2}$ & -1 & 3 & $\frac{11}{2}$ & 9 & 0 & $\frac{417759}{4}$ & 1 & -2 & 4 \\
\hline 16 & -56 & $\frac{126289}{2}$ & 0 & 1 & $\frac{21}{2}$ & 9 & 0 & $\frac{417759}{4}$ & -1 & 2 & 4 \\
\hline 16 & -56 & $\frac{126289}{2}$ & 2 & 0 & 10 & 9 & 0 & $\frac{417759}{4}$ & 0 & 0 & 0 \\
\hline 16 & -56 & $\frac{126289}{2}$ & 3 & -2 & 12 & 9 & 0 & $\frac{417759}{4}$ & 0 & 0 & 6 \\
\hline 16 & -56 & $\frac{126289}{2}$ & 2 & -3 & $\frac{17}{2}$ & 10 & -70 & 112336 & -2 & 0 & 4 \\
\hline 16 & 56 & $\frac{126289}{2}$ & 2 & 1 & $\frac{11}{2}$ & 10 & -70 & 112336 & -1 & 1 & $\frac{5}{2}$ \\
\hline 16 & 56 & $\frac{126289}{2}$ & 0 & -1 & $\frac{3}{2}$ & 10 & -70 & 112336 & 0 & -1 & $\frac{3}{2}$ \\
\hline 16 & 56 & $\frac{126289}{2}$ & 3 & -1 & $\frac{21}{2}$ & 10 & -70 & 112336 & 0 & 2 & 3 \\
\hline 16 & 56 & $\frac{126289}{2}$ & -1 & 1 & $\frac{5}{2}$ & 10 & -70 & 112336 & 1 & 0 & 7 \\
\hline 16 & 56 & $\frac{126289}{2}$ & 0 & 2 & 3 & 10 & -70 & 112336 & 2 & -2 & 7 \\
\hline 16 & 56 & $\frac{126289}{2}$ & 2 & -2 & 7 & 10 & 70 & 112336 & 2 & 0 & 4 \\
\hline 16 & 56 & $\frac{126289}{2}$ & 1 & 0 & 7 & 10 & 70 & 112336 & 1 & -1 & $\frac{5}{2}$ \\
\hline 16 & 56 & $\frac{126289}{2}$ & 1 & 0 & 1 & 10 & 70 & 112336 & 0 & 1 & $\frac{3}{2}$ \\
\hline 16 & 56 & $\frac{126289}{2}$ & -1 & -2 & 4 & 10 & 70 & 112336 & 0 & -2 & 3 \\
\hline 16 & 56 & $\frac{126289}{2}$ & -1 & 1 & $\frac{23}{2}$ & 10 & 70 & 112336 & -1 & 0 & 7 \\
\hline 16 & 56 & $\frac{126289}{2}$ & 1 & -3 & $\frac{11}{2}$ & 10 & 70 & 112336 & -2 & 2 & 7 \\
\hline 16 & 56 & $\frac{126289}{2}$ & 0 & -1 & $\frac{21}{2}$ & 4 & -20 & $\frac{307307}{2}$ & -1 & 0 & 1 \\
\hline 16 & 56 & $\frac{126289}{2}$ & -2 & 0 & 10 & 4 & -20 & $\frac{307307}{2}$ & 0 & 1 & $\frac{3}{2}$ \\
\hline 16 & 56 & $\frac{126289}{2}$ & -3 & 2 & 12 & 4 & -20 & $\frac{307307}{2}$ & 1 & -1 & $\frac{5}{2}$ \\
\hline 16 & 56 & $\frac{126289}{2}$ & -2 & 3 & $\frac{17}{2}$ & 4 & 20 & $\frac{307307}{2}$ & 1 & 0 & 1 \\
\hline 9 & 0 & $\frac{667239}{4}$ & -1 & -1 & $\frac{5}{2}$ & 4 & 20 & $\frac{307307}{2}$ & 0 & -1 & $\frac{3}{2}$ \\
\hline 9 & 0 & $\frac{667239}{4}$ & 1 & 1 & $\frac{5}{2}$ & 4 & 20 & $\frac{307307}{2}$ & -1 & 1 & $\frac{5}{2}$ \\
\hline
\end{tabular}

$C_{2 k}=\Lambda\left(\Phi_{2 k}\right)$ are the Casimir operators of $\mathfrak{s o}(7)$ for the given basis. The decomposition induced by the transformations (10) leads to

$$
\begin{aligned}
& \Phi_{4}=O^{[4,0]}+O^{[2,2]}+O^{[0,4]} \\
& \Phi_{6}=O^{[6,0]}+O^{[4,2]}+O^{[2,4]}+O^{[0,6]} .
\end{aligned}
$$

It follows from this decomposition that we can choose at most three functions that are independent from the invariants of $\mathfrak{s o}(7)$ and the subalgebra. Observe that the operators 
of (43a) and (43b) satisfy the criterion A. Now, computing the Berezin bracket of $O^{[2,2]}$ and $O^{[2,4]}$ gives $\left\{O^{[2,2]}, O^{[2,4]}\right\}=0$; thus by the criterion B it follows at once that

$\left[\Lambda\left(O^{[2,2]}\right), \Lambda\left(O^{[4,2]}\right)\right]=\left[\Lambda\left(O^{[2,2]}\right), \Lambda\left(O^{[2,4]}\right)\right]=\left[\Lambda\left(O^{[4,2]}\right), \Lambda\left(O^{[2,4]}\right)\right]=0$.

The missing label is thus solved by the triplet $\left\{\Lambda\left(O^{[2,2]}\right), \Lambda\left(O^{[4,2]}\right), \Lambda\left(O^{[2,4]}\right)\right\}$. We remark that further decomposing the operator $\Lambda\left(O^{[2,2]}\right)$ with respect to the generators of the different copies of $\mathfrak{s u}(2)$ as in (18) allows us to recover the two commuting operators found in [6], but the procedure applied to the other operators above does not produce a third commuting labelling operator, in accordance with the conclusions of that paper.

In this case, skipping the eigenvalues of the $\mathfrak{s o}(7)$ Casimir operators, an orthonormal basis would e.g. be given by $\left|U, V, W ; \xi_{1}, \xi_{2}, \xi_{3} ; u_{0}, v_{0}, w_{0}\right\rangle$, where $U, V, W$ denote the quadratic Casimir of each copy of $\mathfrak{s u}(2), \xi_{i}$ the eigenvalue of the missing label operators and $u_{0}, v_{0}, w_{0}$ those of the Cartan generators.

\subsection{The $\mathfrak{s u}(6) \supset \mathfrak{s o}(6)$ reduction}

This reduction of $\mathfrak{s u ( 6 )}$ representations to $\mathfrak{s o}(6)$ representations plays an important role in nuclear physics, notably in the interacting boson model [12]. Without considering further reductions and for generic irreducible representations, the number of needed labelling operators is $n=(35-5-15-3) / 2=6$.

We use the standard basis of $\mathfrak{u}(N)$ given by the operators $E_{\mu \nu}, F_{\mu \nu}(1 \leqslant \mu, \nu \leqslant N)$ with the constraints $E_{\mu \nu}+E_{\nu \mu}=0, F_{\mu \nu}-F_{\nu \mu}=0$. The commutation relations over this basis are

$$
\begin{aligned}
& {\left[E_{\mu \nu}, E_{\lambda \sigma}\right]=E_{\nu \sigma}+E_{\lambda \nu}-E_{\mu \sigma}-E_{\lambda \mu}, \quad\left[E_{\mu \nu}, F_{\lambda \sigma}\right]=F_{\nu \sigma}+F_{\lambda \nu}-F_{\mu \sigma}-F_{\lambda \mu},} \\
& {\left[F_{\mu \nu}, F_{\lambda \sigma}\right]=E_{\nu \sigma}+E_{\mu \sigma}-E_{\lambda \mu}-E_{\lambda \nu} .}
\end{aligned}
$$

To recover $\mathfrak{s u}(6)$, we chose the Cartan subalgebra spanned by the vectors $H_{\mu}=F_{\mu \mu}-F_{\mu+1, \mu+1}$ for $\mu=1, \ldots, 5$. Taking into account the isomorphism $\mathfrak{s o}(6) \simeq \mathfrak{s u}(4)$, we take the generators $H_{\mu}, E_{\mu \nu}, F_{\mu \nu}$ with $(1 \leqslant \mu, v \leqslant 4)$ for the subalgebra. A maximal set of independent Casimir invariants is given by the coefficients $\Phi_{k}$ of the characteristic polynomial $\left|\mathrm{i} A_{6}-\lambda \operatorname{Id}_{6}\right|=\lambda^{6}+\sum_{k=2}^{6} C_{k} \lambda^{6-k}$, where $A_{6}$ is the matrix defined by

$$
A_{6}=\left(\begin{array}{cccccc}
-\mathrm{i} Y_{1} & -e_{12}-\mathrm{i} f_{12} & -e_{13}-\mathrm{i} f_{13} & -e_{14}-\mathrm{i} f_{14} & -e_{15}-\mathrm{i} f_{15} & -e_{16}-\mathrm{i} f_{16} \\
e_{12}-\mathrm{i} f_{12} & -\mathrm{i} Y_{2} & -e_{23}-\mathrm{i} f_{23} & -e_{24}-\mathrm{i} f_{24} & -e_{25}-\mathrm{i} f_{25} & -e_{26}-\mathrm{i} f_{26} \\
e_{13}-\mathrm{i} f_{13} & e_{23}-\mathrm{i} f_{23} & -\mathrm{i} Y_{3} & -e_{34}-\mathrm{i} f_{34} & -e_{35}-\mathrm{i} f_{35} & -e_{36}-\mathrm{i} f_{36} \\
e_{14}-\mathrm{i} f_{14} & e_{24}-\mathrm{i} f_{24} & e_{34}-\mathrm{i} f_{34} & -\mathrm{i} Y_{4} & -e_{45}-\mathrm{i} f_{45} & -e_{46}-\mathrm{i} f_{46} \\
e_{15}-\mathrm{i} f_{15} & e_{25}-\mathrm{i} f_{25} & e_{35}-\mathrm{i} f_{35} & e_{45}-\mathrm{i} f_{45} & -\mathrm{i} Y_{5} & -e_{56}-\mathrm{i} f_{56} \\
e_{16}-\mathrm{i} f_{16} & e_{26}-\mathrm{i} f_{26} & e_{36}-\mathrm{i} f_{36} & e_{46}-\mathrm{i} f_{46} & e_{56}-\mathrm{i} f_{56} & -\mathrm{i} Y_{6}
\end{array}\right),
$$

where the vectors $Y_{\mu}$ are given by $Y_{\mu}=\sum_{v=1}^{\mu-1} \frac{-v}{6} h_{v}+\sum_{v=\mu}^{5} \frac{6-v}{6} h_{v}$ [15]. Again, the symmetrization provides the Casimir operators $C_{k}=\Lambda\left(\Phi_{k}\right)$. In this case, the invariants $\Phi_{k}$ decompose as

$$
\begin{aligned}
& \Phi_{3}=O^{[2,1]}+O^{[0,3]} \\
& \Phi_{4}=O^{[4,0]}+O^{[2,2]}+O^{[0,4]} \\
& \Phi_{5}=O^{[4,1]}+O^{[2,3]}+O^{[0,5]} \\
& \Phi_{6}=O^{[6,0]}+O^{[4,2]}+O^{[2,4]}+O^{[0,6]}
\end{aligned}
$$

We observe that following the criterion A, the scalars in each line are mutually commutative. From $(47 a)$ and $(47 b)$ we get at most one labelling operator, which we can choose as $O^{[2,1]}$ 
and $O^{[2,2]}$ without loss of generality. From $(47 c)$ and $(47 d)$ two operators can be taken, respectively. It is easy to verify that the operators $O^{[2,1]}, O^{[2,2]}, O^{[2,3]}$ and $O^{[2,4]}$ are nonfactorizable, and that the following relations hold:

$\left\{O^{[2,1]}, O^{[2,2]}\right\}=\left\{O^{[2,1]}, O^{[2,3]}\right\}=\left\{O^{[2,1]}, O^{[2,4]}\right\}=\left\{O^{[2,2]}, O^{[2,3]}\right\}=\left\{O^{[2,2]}, O^{[2,4]}\right\}=0$.

As a consequence, their symmetrizations $\Theta^{[p, q]}=\Lambda\left(O^{[p, q]}\right)$ commute. From the criterion $\mathrm{B}$ we further obtain that $\Theta^{[2,1]}$ and $\Theta^{[2,2]}$ commute with $\Theta^{[4,1]}, \Theta^{[0,5]}, \Theta^{[4,2]}$ and $\Theta^{[0,6]}$, respectively. Choosing for example the operators $\left\{\Theta^{[2,1]}, \Theta^{[2,2]}, \Theta^{[4,1]}, \Theta^{[2,3]}, \Theta^{[4,2]}, \Theta^{[2,4]}\right\}$, we get the required set of labelling operators. We remark that the criterion $\mathrm{B}$ simplifies the problem, reducing the computation of Berezin brackets from 15 to only 5 .

For the chain $\mathfrak{s u ( 6 )} \supset \mathfrak{s o ( 6 )}$ and a generic IR, we need 15 labels to unambiguously separate the states. While the Casimir operators of $\mathfrak{s o}(6)$ and the preceding missing label operators provide nine of these labels, the six remaining ones must be internal $\mathfrak{s o}(6)$ operators. Again, using the isomorphism $\mathfrak{s o}(6) \simeq \mathfrak{s u}(4)$, these internal operators can be taken from the subalgebra chain

$$
\mathfrak{s u}(4) \supset \mathfrak{s u}(3) \supset \mathfrak{s u}(2) \supset \mathfrak{s o}(2) \text {. }
$$

A possible choice would be given by $H_{1}, H_{2}, H_{3}$ and the Casimir operators of $\mathfrak{s u}(3)$ and $\mathfrak{s u}(2)$.

\section{4. $\mathfrak{s o}(7) \supset \mathfrak{s o}(5)$}

As a typical example of a labelling problem that cannot be solved completely with the decomposition, but requires additional subgroup scalars, we consider the chain $\mathfrak{s o}(7) \supset \mathfrak{s o}(5)$ with $n=3$ labelling operators [7, 16].

Taking the basis consisting of the operators $G_{a}^{b}$ with $-3 \leqslant a, b \leqslant 3$ and constraints $G_{b}^{a}+G_{-a}^{-b}=0$, the brackets are given by

$$
\left[G_{b}^{a}, G_{d}^{c}\right]=\delta_{b}^{c} G_{d}^{a}-\delta_{d}^{a} G_{b}^{c}+\delta_{d}^{-b} G_{-a}^{c}-\delta_{-a}^{c} G_{d}^{-b},
$$

the subalgebra being generated by the $G_{a}^{b}$ with $-2 \leqslant a, b \leqslant 2$. With respect to the corresponding transformations (10), the Casimir operators of $\mathfrak{s o}(7)$ (see e.g. [7, 17]) decompose as

$$
C_{4}=\Theta^{[4,0]}+\Theta^{[2,2]}+\Theta^{[1,3]}+\Theta^{[0,4]}, \quad C_{6}=\Theta^{[4,2]}+\Theta^{[3,3]}+\Theta^{[2,4]},
$$

where $\Theta^{[4,0]}$ corresponds to the quartic Casimir operator of $\mathfrak{s o}(5)$. Observe that criteria $A$ and $\mathrm{B}$ cannot be applied to this case. Computing the Berezin bracket for the projections $O^{[a, b]}=\pi\left(\Theta^{[a, b]}\right)$ of these operators, we find that only the following are in involution:

$$
\left\{O^{[2,2]}, O^{[4,2]}\right\}=0, \quad\left\{O^{[1,3]}, O^{[3,3]}\right\}=0, \quad\left\{O^{[0,4]}, O^{[2,4]}\right\}=0 .
$$

Thus, the decomposition of Casimir operators does not suffice to solve the MLP, and step (vi) of the algorithm must be applied. Looking for scalars having order 3 , we find the three operators $O^{[2,1]_{a}}, O^{[2,1]_{b}}$ and $O^{[1,2]}$. Computing the Berezin bracket of these operators with the pair in involution above, it turns out that only one of the scalars commutes with them:

$$
\left\{O^{[1,2]}, O^{[1,3]}\right\}=0, \quad\left\{O^{[1,2]}, O^{[3,3]}\right\}=0 .
$$

To check the independence of the operators $\left\{O^{[1,2]}, O^{[1,3]}, O^{[3,3]}\right\}$ with the Casimir operators of the Lie algebras, we compute the following Jacobian:

$$
\frac{\partial\left\{\pi\left(C_{2}\right), \pi\left(C_{4}\right), \pi\left(C_{6}\right), O^{[2,0]}, O^{[4,0]}, O^{[1,2]}, O^{[1,3]}, O^{[3,3]}\right\}}{\partial\left(g_{-3}^{2}, g_{-3}^{1}, g_{-3}^{0}, g_{-2}^{1}, g_{-2}^{0}, g_{-1}^{1}, g_{1}^{2}, g_{1}^{3}\right)} \neq 0 .
$$

As a consequence, a possible choice of subgroup scalars to solve this labelling problem is given by the symmetrized operators $\left\{\Theta^{[1,2]}, \Theta^{[1,3]}, \Theta^{[3,3]}\right\}$. 
For this reduction, the nine labels required for an orthonormal basis of states can be taken as

$$
\left|\lambda_{1}, \lambda_{2} ; \xi_{1}, \xi_{2}, \xi_{3} ; a_{1}, a_{2}, b_{1}, b_{2}\right\rangle
$$

where $\lambda_{i}$ correspond to eigenvalues of the $\mathfrak{s o}(5)$ Casimir operators, $\xi_{i}$ to those of the missing label operators, $a_{1}$ and $a_{2}$ to the value of $G_{1}^{1}$ and $G_{2}^{2}$, respectively, and $b_{i}$ denotes the eigenvalue of $\left(G_{i}^{i}\right)^{2}+G_{0}^{i} G_{i}^{0}+G_{i}^{0} G_{0}^{i}$ for $i=1,2$.

\section{Summary and outlook}

Combining the decomposition of Casimir operators induced by the embedding of a subalgebra with the analytical expression for the commutator of labelling operators and their properties, especially the two new criteria deduced from them, we have described an analytical algorithm in six steps to solve the internal labelling problem. This procedure has the advantage of avoiding the usually complicated manipulations of elements in the enveloping algebras of Lie algebras. In most cases, the algorithm will provide only $n$ of the $2 n$ available operators to solve the MLP, specially for those solved using only steps (i)-(v). This is a consequence of the well-known division of labelling operators into two disjoint sets with respect to the commutativity of operators [2]. However, in some special cases even an integrity basis can be deduced, as happens for example for the chain $\mathfrak{s o ( 7 )} \supset G_{2}$ with $n=1$ label, where the decomposition of the Casimir operators provide the two operators required to determine an integrity basis [2]. Labelling problems that usually require step (vi) of the algorithm are those related to the embedding of principal subalgebras [18].

The algorithm cannot be applied in general beyond reduction chains of reductive Lie algebras for various reasons. The failure of complete reducibility of representations is the first, another being the possible non-existence of polynomial invariants for the Lie algebras [8].

We have also proposed a practical receipt to construct recursively an orthonormal basis of eigenstates for a given IR of $\mathfrak{s}$. The main idea of this explicit basis is to first diagonalize the Casimir operators of the subalgebra, in order to have the eigenspace decomposition induced by the branching rules, and then to diagonalize the missing label operators, to separate multiplicities, and finally to diagonalize the internal subgroup operators. We remark that this receipt is only one of the various different possibilities to determine a basis of eigenstates.

An interesting question that arises naturally from the algorithm and the basis construction concerns the labelling problem for degenerate representations. In this case, some of the labels may not define states for degenerate IRs of interest, therefore reducing the number of required labelling operators [19]. It is natural to ask whether there is some possible refinement of the algorithm that describes the polynomial relations satisfied by the generators when acting on the states of a degenerate representation, or if the difficult analysis of generating functions is unavoidable [20]. However, how this ansatz should be made is still unclear.

For labelling problems of the form $\mathfrak{s} \supset \mathfrak{s}^{\prime} \supset \cdots \supset \mathfrak{s}^{(n)}$ the proposed scheme should also be useful, as it allows us to combine subgroup scalars in any reduction step with the successive decomposition of Casimir operators in each of the subalgebras. Suitable and physically significant labelling problems exhibiting this structure are, for example, those involving the angular momentum algebra $\mathfrak{s o ( 3 ) . ~ O t h e r ~ p h y s i c a l l y ~ r e l e v a n t ~ l a b e l l i n g ~ p r o b l e m s ~ w h e r e ~}$ the algorithm could be of application are those related to exceptional groups, especially the $E$-series used in high energy physics [21,22]. The main difficulty for these cases lies in the fact that the existing formulae for the Casimir operators of $E_{6}, E_{7}$ and $E_{8}$ are rather formal, based on special bases of their complexification, and that no simple analytical approach to compute 
them over arbitrary bases is known. In this sense, a first step towards the systematization of labelling problems with exceptional algebras consists of developing practical procedures to compute their invariants in either an analytical or geometrical frame [23]. Currently efforts in this direction are in progress.

\section{Acknowledgments}

The referees are acknowledged for suggesting subsection 4.1 and various improvements of the manuscript. During the preparation of this work, the author was financially supported by the research project GR58/4120818-920920 of the UCM-BSCH.

\section{References}

[1] Elliott J P 1958 Proc. R. Soc. A 245 128, 562

Jarvis P D 1974 J. Phys. A: Math. Nucl. Gen. 71807

Rowe D J 1994 J. Math. Phys. 353178

Hecht K T 1994 J. Phys. A: Math. Gen. 273445

Rowe D J 1995 J. Math. Phys. 361520

[2] Sharp R T and Pieper S C 1968 J. Math. Phys. 9663

Sharp R T 1970 Proc. Camb. Phil. Soc. 68571

Sharp R T 1975 J. Math. Phys. 162050

Gaskell R, Rosensteel G and Sharp R T 1981 J. Math. Phys. 222732

[3] Jarvis P D 1979 J. Phys. A: Math. Gen. 121

Jarvis P D and Zhang R B 2005 J. Phys. A: Math. Gen. 38 L219

[4] Boya L J and Campoamor-Stursberg R 2009 J. Phys. A: Math. Theor. 42205235

[5] Campoamor-Stursberg R 2008 J. Phys. Conf. Ser. 128012052

Campoamor-Stursberg R 2008 arXiv:0805.2981 [hep-th]

[6] Van der Jeugt 1984 J. Math. Phys. 251221

[7] Bincer A M 1983 J. Math. Phys. 241695

[8] Peccia A and Sharp R T 1976 J. Math. Phys. 171313

Pecina Cruz J N 1994 J. Math. Phys. 353146

[9] Campoamor-Stursberg R 2007 J. Phys. A: Math. Theor. 4014773

[10] Judd B R, Miller W, Patera J and Winternitz P 1974 J. Math. Phys. 151787

[11] Berezin F A 1967 Funkt. Anal. Prilozh. 11

[12] Iachello F 2006 Lie Algebras and Applications (New York: Springer)

[13] Pan F, Cao Y-F and Pan Z-Y 1989 J. Phys. A: Math. Gen. 224105

[14] Bincer A M and Riesselmann K 1993 J. Math. Phys. 345935

[15] Campoamor-Stursberg R 2005 J. Phys. A: Math. Gen. 384187

[16] Racah G 1951 Group Theory and Spectroscopy (Princeton, NJ: Princeton University Press)

[17] Gel'fand I M 1950 Mat. Sb. 26112

[18] De Meyer H, Vanden Berghe G, Van der Jeugt J and De Wilde P 1985 J. Math. Phys. 262124 De Baerdemacker S, Heyde K and Hellemans V 2007 J. Phys. A: Math. Theor. 402733

Campoamor-Stursberg R 2009 J. Phys. Conf. Ser. 175012008

[19] Giroux Y and Sharp R T 1987 J. Math. Phys. 281671

[20] Couture M and Sharp R T 1989 J. Phys. A: Math. Gen. 221525

[21] Wybourne B G and Bowick M J 1977 Aust. J. Phys. 30259 Slansky R 1981 Phys. Rep. 791

[22] Wybourne B G 1995 Lithuanian J. Phys. 35123 Keurentjes A 2003 Nucl. Phys. B 658303

[23] Boyko V, Patera J and Popovych R 2006 J. Phys. A: Math. Gen. 395749 Boyko V, Patera J and Popovych R 2007 J. Phys. A: Math. Theor. 40113 